\title{
Zur Problematik der realistischen Beurteilung und Quantifizierung der Materialparameter für numerische Simulationen
}

\author{
Tobias Ladinig
}

Lehrstuhl für Bergbaukunde, Bergtechnik und Bergwirtschaft, Montanuniversität Leoben, Leoben, Österreich

Eingegangen 26. August 2019; angenommen 29. August 2019; online publiziert 10. September 2019

\begin{abstract}
Zusammenfassung: Numerische Simulationen finden eine breite Anwendung in der gebirgsmechanischen Planung. Kritisch für die Aussagekraft des Ergebnisses ist die Qualität der Eingangsparameter. Die Materialparameter von Gebirge nehmen dabei eine besondere Stellung ein, weil die Erfassung und Beurteilung der Gebirgseigenschaften im Allgemeinen nicht ohne Annahmen und Abschätzungen möglich ist. Daraus folgt, dass die Materialparameter des Gebirges Unsicherheiten und Streubreiten aufweisen. Dieser Umstand wird anhand von einfachen und praktischen Beispielen diskutiert. Abschließend werden Möglichkeiten zum Umgang mit diesen Unsicherheiten besprochen. Diese sind Erfahrung aus dem fortschreitenden Abbau, das Prinzip der kontinuierlichen Planung und das Ausweisen einer Versagenswahrscheinlichkeit.
\end{abstract}

Schlüsselwörter: Gebirgsmechanik, Gebirgsklassifikation, Gebirgseigenschaften, Numerische Simulation

Considerations Related to a Realistic Assessment and Quantification of Material Properties for Numerical Simulations

Abstract: Numerical simulations are widely applied in the rock mechanical design. The quality of the result depends on the realistic assessment of input parameters strongly. As usually assumptions and estimations are required in the determination of rock mass parameters, they are associated with uncertainty. Some illustrative and practical examples are shown to highlight this issue. Approaches to deal with such uncertainties are outlined. They include the experience gained in ongoing mining activities, the princi-

\footnotetext{
Dipl.-Ing. T. Ladinig ( $\bowtie)$

Lehrstuhl für Bergbaukunde, Bergtechnik und Bergwirtschaft,

Montanuniversität Leoben,

Franz-Josef-Straße 18,

8700 Leoben, Österreich

tobias.ladinig@unileoben.ac.at
}

ple of continuous design and the calculation of a probability of failure.

Keywords: Rock mechanics, Rock mass classification, Rock mass properties, Numerical simulations

\section{Einleitung}

Numerische Simulationen werden neben empirischen, semi-empirischen und analytischen Verfahren zur gebirgsmechanischen Planung im Bergbau eingesetzt. Dabei werden sie für eine Vielzahl an Fragestellungen herangezogen, jedoch insbesondere zur Untersuchung von komplexen Zusammenhängen. Beispiele dafür sind unter anderem Stabilitätsanalysen von Tagebauböschungen oder untertägigen Hohlräumen, Rückanalysen und Fallstudien spezifischer Aspekte oder die Beurteilung des Einflusses von Abbausequenzen. Obwohl die angeführten Anwendungsgebiete unterschiedliche Themenfelder betreffen, haben sie gemeinsam, dass jede numerische Simulation eine entsprechende Planung benötigt. In dieser Vorbereitungsphase muss zunächst geklärt werden, welche Situation analysiert wird und wie diese Situation im Detail aussieht. Die Details umfassen die Geometrie, Gesteins- und Gebirgsbedingungen sowie Überlegungen zum voraussichtlichen Versagensmechanismus. Diese Information wird in weiterer Folge benötigt, um das Simulationsverfahren, Simulationsmodell und weitere Details auszuwählen. Nach Festlegung der Simulationsdetails muss definiert werden, welche Eingangsparameter benötigt und wie diese bestimmt werden. Zusammengefasst kann festgehalten werden, dass verschiedene Situationen einen unterschiedlichen Aufbau der Simulation verlangen. Beispielhaft dafür ist die Berechnung des Sicherheitsfaktors einer Tagebauendböschung bzw. die Betrachtung eines Keilversagens in einer Bruchwand. Für erstere Berechnung sind die Materialeigenschaften des Gesteins- bzw. Gebirgsverbands üblicherweise relevanter, während für das Keilversagen 
die Eigenschaften und Orientierung der Trennflächen als kritischer einzustufen sind.

\section{Eingangsparameter}

Die Eingangsparameter in Simulationen umfassen zumeist Gesteins- und Gebirgsparameter, welche auch als Materialparameter bezeichnet werden können, sowie die primäre Spannungssituation. Aufgrund der diskontinuierlichen und großräumigen Natur des Gebirges ist es im Allgemeinen nicht möglich, die Materialparameter direkt zu bestimmen. Zudem variieren die Gesteins- und Gebirgseigenschaften oftmals über kurze Distanzen. Diese Besonderheiten müssen in der Ableitung der Materialparameter berücksichtigt werden. Nachfolgend sind Beispiele angeführt.

\subsection{Gestein und Gebirge}

Gestein als die "kleinste Einheit“ kann in vielen Fällen als annähernd homogen beschrieben werden. Die Gesteinseigenschaften werden heutzutage üblicherweise anhand von Laborversuchen bestimmt. Weitere Möglichkeiten stellen In-situ-Versuche oder die indirekte Bestimmung aus Tabellen dar. Im Gegensatz zum Gestein ist Gebirge grundsätzlich inhomogen. Es ist ein komplexes System bestehend aus Gestein, Trennflächen und Wasser und weist im Allgemeinen deutlich geringere Festigkeiten als Gestein auf. Die Bestimmung von Gebirgseigenschaften an Hand von großmaßstäblichen Labor- oder In-situ-Versuchen ist aufgrund des notwendigen Aufwands für einen regelmäßigen Einsatz ungeeignet und grundsätzlich Forschungszwecken vorbehalten. Vielmehr erfolgt die Quantifizierung der Gebirgseigenschaften auf der Basis von Gebirgsklassifikationssystemen. Das Gebirge wird hierbei indirekt über die Gesteinseigenschaften, die Trennflächenstruktur, die Trennflächeneigenschaften, die Bergwassersituation sowie weitere Parameter beschrieben. Basierend auf der Gebirgsklassifikation können die Materialparameter des Gebirges abgeleitet werden.

\subsection{Beschreibung der Trennflächenstruktur}

Die Trennflächenstruktur beschreibt die räumliche Verteilung der Trennflächen und ist generell dreidimensional. In die Struktur fließen Anzahl, Abstand sowie die Orientierung von Trennflächen ein. Zur Beschreibung der Trennflächenstruktur greifen die Gebirgsklassifikationssysteme auf Bohrkernauswertungen sowie In-situ-Aufnahmen zurück. Die Erfassung der Dreidimensionalität der Trennflächenstruktur ist mit den derzeit eingesetzten Methoden schwierig und nur bedingt möglich.

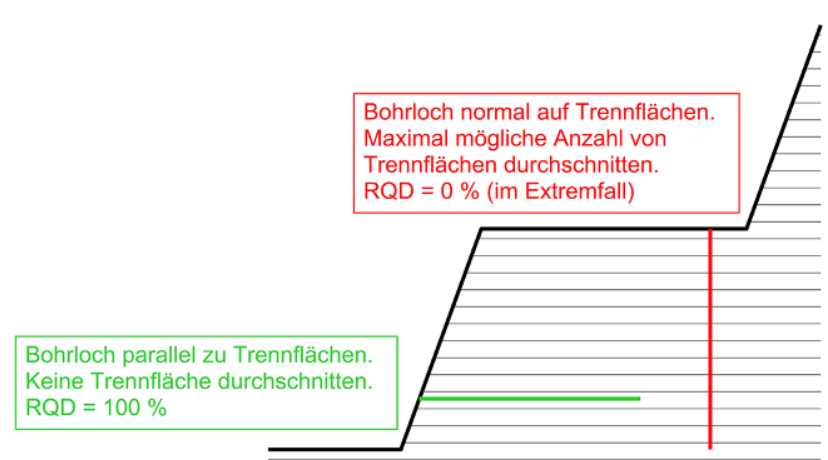

Abb. 1: Schematische Darstellung der Richtungsabhängigkeit des RQD

\subsubsection{Rock Quality Designation (ROD)}

Der ROD nach Deere [1] fließt in mehrere Klassifikationssysteme ein. Er ist ein Maß für den Zerlegungsgrad bzw. die Klüftigkeit des Gebirges und errechnet sich nach GI. 1.

$$
R Q D=\frac{\sum(\text { Kernstücke }>10 \mathrm{~cm})}{\text { Gesamtkernlänge }} * 100[\%]
$$

Der RQD ist ein eindimensionaler Wert, weil lediglich die Trennflächenschnittpunkte entlang der Mittellinie des Bohrkerns betrachtet werden. Die Orientierung der Trennflächen bleibt unberücksichtigt. Diese Eindimensionalität und Richtungsabhängigkeit des ROD ist schematisch in Abb. 1 dargestellt. In der gezeigten Bruchwand liegt ein Trennflächensystem vor. Abhängig von der Richtung der Kernbohrung kann der RQD die Extremwerte von 100\% bzw. $0 \%$ annehmen. Daraus kann geschlossen werden, dass der ROD die Räumlichkeit der Trennflächenstruktur nur bedingt beschreibt. In den meisten Gebirgsklassifikationssystemen wird das Trennflächengefüge daher noch mit weiteren Parametern, beispielsweise dem durchschnittlichen Trennflächenabstand, der Trennflächendurchgängigkeit oder der Anzahl der Trennflächensysteme, erfasst.

\subsubsection{Ortsaufnahmen}

Ortsaufnahmen dienen unter anderem zur Erfassung der Anzahl der Trennflächensysteme, des Trennflächenabstands und der Trennflächendurchgängigkeit. Die Aufnahmen erfolgen zumeist entlang eines Streckenulms oder einer Bruchwand, welche geometrisch betrachtet Ebenen sind. Diese Aufnahmen sind im Allgemeinen zweidimensional und nur teilweise dreidimensional. Denn Trennflächen bzw. Trennflächensysteme, die einen flachen Winkel zur Aufnahmeebene einschließen, werden entweder nur schwer oder nicht erfasst; vgl. Abb. 2. Dieselbe Überlegung gilt für die Ermittlung des Trennflächenabstands, welcher der Normalabstand zwischen benachbarten Trennflächen ist.

Zusammengefasst kann festgehalten werden, dass zur adäquaten Beschreibung des Gebirges anhand von Gebirgsklassifikationen sorgfältige und genaue Aufnahmen notwendig sind. Dennoch beruhen die Aufnahmen zum Teil auf Annahmen und subjektiven Abschätzungen und sind daher mit Unsicherheiten behaftet. Demgemäß muss 


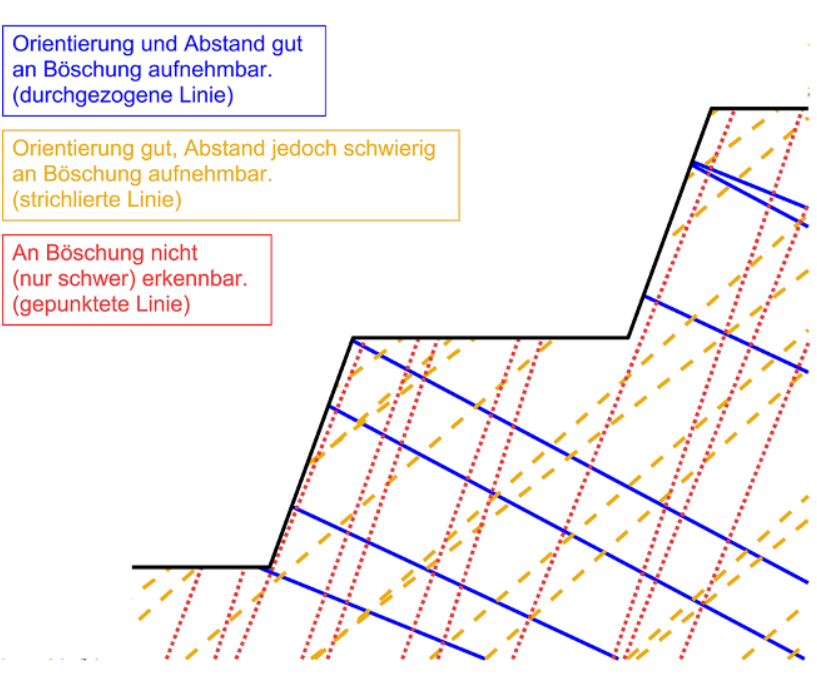

Abb. 2: Schematische Darstellung zur Erkennbarkeit von Trennflächensystemen

das Ergebnis einer Gebirgsklassifikation eine Bandbreite der Werte beinhalten. Die Angabe eines einzelnen, durchschnittlichen Werts beschreibt die Gebirgsbedingungen unzureichend.

\subsection{Ableitung der Materialparameter des Gebirges}

\subsubsection{Gebirgsbeschreibung und Gebirgsklassifi- kation}

Aufbauend auf Ergebnissen einer Gebirgsklassifikation werden Gebirgseigenschaften, wie die Festigkeit und der E-Modul, abgeleitet. Dazu kann auf Tabellenwerte und Formeln aus der Literatur zurückgegriffen werden. Dabei muss unbedingt die Anwendbarkeit und Gültigkeit der angewandten Formeln bzw. Bruchgesetze für die vorliegenden Gebirgsformationen und Aufgabenstellungen überprüft werden. Das Bruchgesetz nach Hoek-Brown ist beispielsweise nicht zur Beschreibung eines Gebirges mit einzelnen diskreten Trennflächen geeignet. Zudem sind die abgeleiteten Materialparameter kritisch zu betrachten und den Abbauerfahrungen gegenüberzustellen. Durch diese Maßnahme lässt sich die Plausibilität der Gebirgseigenschaften überprüfen [2]. Bei Abweichungen vom erwarteten Gebirgsverhalten können Rückanalysen zur Parameterbestimmung eingesetzt werden.

\subsubsection{Abbauerfahrung, Rückanalyse und Versagensfälle}

Der Einsatz eines gebirgsmechanischen Monitoringprogramms ermöglicht eine möglichst objektive Erfassung von Abbauauswirkungen im voranschreitenden Abbau. Die systematische Rückanalyse dieser dokumentierten Abbauauswirkungen ermöglicht eine Ableitung von Gebirgseigenschaften und Aussagen betreffend Gebirgsverhalten. Beobachtungen umfassen beispielsweise Zustände von Hohlräumen und Bruchwänden sowie gemessene
Abb. 3: Abgleiten eines Felskeils entlang einer Kluftfläche

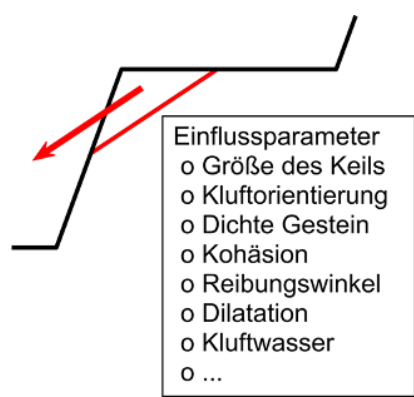

Verschiebungen. Oftmals wird die Rückanalyse mit einer Gebirgsklassifikation kombiniert.

Die Ableitung von Materialparametern aus Versagensfällen stellt eine Form der Rückanalyse dar. Poisel empfiehlt die Bevorzugung der Ableitung von Materialparametern für Böschungsberechnungen aus Rückrechnung abgeglittener Felspartien gegenüber Gebirgsklassifikationen und Laborversuchen [3]. Diese Empfehlung wird nachfolgend anhand eines einfachen Beispiels, dem Abgleiten eines Keils entlang einer ebenen Kluftfläche, in einer zweidimensionalen Betrachtung diskutiert. Abb. 3 stellt die Situation schematisch dar.

Bei Beschreibung der Kluftfestigkeit über das Bruchgesetz nach Mohr-Coulomb müssen über die Rückanalyse die Kohäsion und der Reibungswinkel bestimmt werden. Die angreifende Gewichtskraft kann über das Blockvolumen und die Dichte ausreichend genau berechnet werden. Da im betrachteten Fall aus einem Versagen zwei Parameter ermittelt werden sollen, muss einer der beiden Parameter angenommen werden, damit der andere berechnet werden kann. Durch das Betrachten mehrerer gleichartiger Versagensfälle kann diesem Umstand begegnet werden. Jedoch ist an dieser Stelle festzuhalten, dass das Beispiel ein äußerst einfacher und idealisierter Fall ist. In der Realität werden unter anderem zumeist zwei oder mehr (unebene) Trennflächen, Kluftwasser und variierende Festigkeitseigenschaften involviert sein.

Das obige Beispiel illustriert, dass bereits in einem einfachen Versagensfall Annahmen notwendig werden. In deutlich komplexeren Fällen, beispielsweise dem Versagen einer Tagebauendböschung entlang eines Gleitkreises, steigt die Anzahl der Annahmen signifikant an. Offen ist zudem, wie die Materialparameter für die Dimensionierung einer Tagebauendböschung aus der Rückrechnung eines Versagensfalls in der vorliegenden Gebirgssituation abgeleitet werden können.

Versagensfälle können für die Bestimmung von Materialparametern wertvolle Beiträge leisten, jedoch ist die Anzahl der zu analysierenden Fälle gering. Hier bieten Rückanalysen aus anderweitigen Abbaubeobachtungen den Vorteil, dass unterschiedliche Situationen und eine größere Anzahl von Fällen betrachtet werden können. Dies führt zu einer Reduktion der Unsicherheiten in der Materialparameterbestimmung. 


\subsection{Räumliche Variation der Gebirgs- eigenschaften}

Die Gebirgseigenschaften weisen grundsätzlich eine gewisse Streubreite auf und variieren räumlich. In numerischen Simulationen werden diese lokalen Variationen und die Streuung der Eigenschaften oftmals vernachlässigt. Zudem wird das (inhomogene) Gebirge zur Vereinfachung in vielen Fällen als homogen modelliert.

\section{Umgang mit Unsicherheiten}

Aus den angeführten Überlegungen und Beispielen ist ersichtlich, dass die Materialparameter mit einer gewissen Unsicherheit sowie Streubreite verbunden sind. Dieser Umstand liegt in der Natur des Bergbaus und der Gebirgsmechanik. Ein entsprechender Umgang mit Unsicherheiten und Streubreiten von Materialparametern ist daher unabdinglich. Möglichkeiten zum Umgang werden nachfolgend angeführt.

\subsection{Abbauerfahrung und kontinuierliches Design}

Bergbaubetriebe können über mehrere Jahrzehnte bestehen. Durch den Abbau werden fortlaufend Erfahrungen betreffend Gebirgsverhalten gesammelt. Dazu wird der Einsatz eines kontinuierlichen, zielgerichteten und ausgewogenen Monitoringprogramms empfohlen. Zudem kann der Zuschnitt durch den Einsatz eines kontinuierlichen Designund Optimierungsprozesses an die gewonnenen Erkenntnisse und eventuell veränderte Gebirgsbedingungen angepasst werden (vgl. Abb. 4). Dadurch wird eine gewisse Flexibilität betreffend Entscheidungen im Zuschnitt, welche die regionale (Langzeit-)Stabilität (z. B. Generalneigung und Orientierung einer Tagebauendböschung) beeinflussen, bis zu einem fortgeschrittenen Abbaustadium erhalten.

Die obigen Punkte stellen einen wesentlichen Unterschied zum zivilen Ingenieurbau dar, bei welchem der Endzustand in den meisten Fällen zu Beginn geplant und realisiert wird

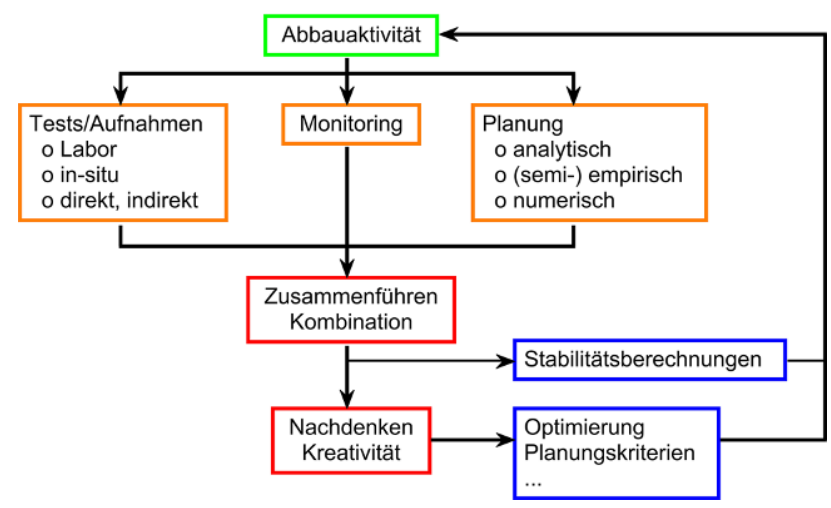

Abb. 4: Darstellung des kontinuierlichen Designprozesses

\subsection{Versagenswahrscheinlichkeit}

Der Sicherheitsfaktor ist definiert als der Quotient von Festigkeit und Belastung; vgl. GI. 2. Er wird zur Planung von Strukturen wie Tagebauböschungen, Abbaufesten oder Ausbausystemen eingesetzt. Dabei bedeutet ein Sicherheitsfaktor von $\geq 1$, dass die betrachtete Struktur stabil sein sollte und ein Sicherheitsfaktor $<1$, dass ein Versagen eintreten sollte.

$$
\text { Sicherheitsfaktor }=\frac{\text { Festigkeit }}{\text { Belastung }}[-]
$$

Sowohl die Festigkeit als auch die Belastung einer Struktur beinhalten Unsicherheiten. In diesen Fällen kann der Sicherheitsfaktor mit Mittelwerten berechnet werden. Aufgrund der Streubreite der Parameter kann dennoch bei einem Sicherheitsfaktor $\geq 1$ ein Versagen eintreten. Umgekehrt können Strukturen mit einem Sicherheitsfaktor $<1$ stabil sein. Daher sollen Sicherheitsfaktoren in Kombination mit einer Versagenswahrscheinlichkeit betrachtet werden. Die Versagenswahrscheinlichkeit kann beispielsweise über eine Parametervariation oder Monte-Carlo-Simulation berechnet werden. Sie ist im Vergleich zum Sicherheitsfaktor deutlich aussagekräftiger und erlaubt eine Abschätzung des Risikos sowie die Einrichtung eines darauf aufbauenden Risikomanagements.

\section{Schlussfolgerung}

Numerische Simulationen sind ein mögliches Verfahren in der gebirgsmechanischen Bergbauplanung. Sie ermöglichen eine Erfassung und Beschreibung von komplexen Zusammenhängen, welche in vielen gebirgsmechanischen Fragestellungen auftreten. Eine realistische Beurteilung der Material- und weiteren Eingangsparameter ist für die Qualität und Aussagekraft der Simulation entscheidend. Die inhomogene und diskontinuierliche Natur des Gebirges bedingt, dass dessen Materialparameter nicht exakt bestimmt werden können und gewisse Unsicherheiten und Streubreiten aufweisen. Der Einsatz einer kontinuierlichen Planung und Optimierung sowie eines Monitoringprogramms zur Beobachtung der Abbauauswirkungen ermöglicht einen adäquaten Umgang mit diesen Unsicherheiten. Des Weiteren empfiehlt sich, Standsicherheiten auf Basis von Sicherheitsfaktor und Versagenswahrscheinlichkeit zu beurteilen und darauf aufbauend ein Risikomanagementprogramm zu implementieren. Begünstigend wirkt sich zudem der Umstand aus, dass Bergbau ein dynamisches System ist, und Entscheidungen betreffend regionale und langfristige Standfestigkeit im Allgemeinen erst in einem fortgeschrittenen Abbaustand, in welchem bereits Erfahrungen bezüglich Gebirgseigenschaften und Gebirgsverhalten vorhanden sind, getroffen werden.

Funding. Open access funding provided by Montanuniversität Leoben.

Open Access Dieser Artikel wird unter der Creative Commons Namensnennung 4.0 International Lizenz (http://creativecommons.org/licenses/ by/4.0/deed.de) veröffentlicht, welche die Nutzung, Vervielfältigung, Bearbeitung, Verbreitung und Wiedergabe in jeglichem Medium und For- 
mat erlaubt, sofern Sie den/die ursprünglichen Autor(en) und die Quelle ordnungsgemäß nennen, einen Link zur Creative Commons Lizenz beifügen und angeben, ob Änderungen vorgenommen wurden.

\section{Literatur}

1. Deere, D.U.: Technical description of rock cores for engineering purposes, Rock mechanics and engineering geology, vol. 1 (1964), no. 1, pp 17-22

2. Ladinig, T.; Wagner, $\mathrm{H}$.; Daborer, A.: Effect of stoping activities on tunnel conditions in a deep, hard rock mine in Austria, in: Joug hin W. (ed.): Proceedings of the Ninth International Conference on Deep and High Stress Mining, , Johannesburg, South Africa: The Southern African Institute of Mining and Metallurgy, 2019, pp 87-96 3. Poisel, R.: Felsstürze - Ablösemechanismen, in: Poisel, R.; Preh, A.; Kolenprat, B. (Hrsg.): Tagungsband - Gefahren durch Steinfall und Felssturz, Wien: Geologische Bundesanstalt, 2018, S. 49-58

Hinweis des Verlags. Der Verlag bleibt in Hinblick auf geografische Zuordnungen und Gebietsbezeichnungen in veröffentlichten Karten und Institutsadressen neutral. 